\title{
Situación de la brecha digital de género $y$ medidas de inclusión en España
}

\author{
Mercedes Caridad Sebastian * \\ Maria Dolores Ayuso García **
}

Artículo recibido:

12 de abril de 2011.

Artículo aceptado:

16 de enero de 2012.

\section{RESUMEN}

La presente investigación establece en el contexto económico y social mundial, la importancia que tiene el desarrollo de las TIC y las notables diferencias que existen en cuanto acceso, uso, penetración y habilidades, resultado de otras brechas sociales, incluida la brecha de género objeto de nuestra investigación. Se analizan los datos estadísticos sobre la Sociedad de la Información con indicación de género en la UE de los 15 y la de los 27, y en España. Por último se analizan las medidas normativas y legislativas de carácter general y horizontal acometidas por los poderes públicos para evitar las desigualdades, esto es, la exclusión, y se

* Mercedes Caridad Sebastian. Universidad Carlos III de Madrid, España. mercedes@bib.uc3m

** Maria Dolores Ayuso García. Universidad de Murcia, España. mayu@um.es

INVESTIGACIÓN BIBLIOTECOLÓGICA, Vol. 25, Núm. 55, septiembre/diciembre, 2011, México, ISSN: 0187-358X. pp. 227-252 
estudian los planes específicos para la inclusión digital de género que se llevan a cabo en las CCAA y cuáles de estos son más beneficiosos y se ajustan mejor a las necesidades que se demandan. Se concluye que tal brecha es más profunda en las categorías de usuarios avanzados y muy avanzados que utilizan con destreza herramientas de software y que es necesario revisar los indicadores de medición.

Palabras clave: Sociedad de la información; Brecha digital de género; Medidas de inclusión en España; Planes de e-inclusión en las CCAA; Brecha digital de género en España; Acciones de inclusión en la UE.

ABSTRACT

Status of the gender digital divide and inclusion measures in Spain

Mercedes Caridad Sebastian and Maria Dolores Ayuso García

This research establishes, in a global economic and social context, the importance of ICT development, and the remarkable differences in access, use, insight and skills, as a result of other social gaps, such as the gender gap we focused on in our study. The statistical data on the Information Society in EU 15 and EU 27 in Spain are examined with an indication of gender. Finally, the general policy and legislative measures undertaken by governments to prevent inequalities, that is, exclusion, are revised. We also examine the specific plans for gender digital inclusion that take place in the Spanish Autonomous Communities and which of these are more beneficial and more suitable to the needs that are demanded. We conclude that the digital divide of gender is deeper in the categories of advanced and very advanced users, that use software tools skillfully, and in the need to review the indicators.

Keywords: Information Society; Gender digital divide, Inclusion measures in Spain; E-inclusion plans in Spanish Autonomous Communities; Gender digital divide in Spain; Inclusion actions in the EU. 


\section{INTRODUCCIÓN}

— n los últimos diez años del presente siglo, uno de los rasgos esenciales Edel desarrollo económico y social en los países de la Unión Europea (UE) y de la Organización para la Cooperación y el Desarrollo Económico [OCDE, 2006] ha sido el acceso, uso, intensidad y habilidades relacionadas con las Tecnologías de la Información y la Comunicación (TIC) en la Sociedad de la Información (SI) [Caridad, Ayuso y Ayuso, 2010]. No olvidemos, por otra parte, a la Unesco [2010] promotora de la dimensión social de la SI como elemento clave de la Sociedad del Conocimiento. Estas condiciones sociales, económicas, tecnológicas, etc. se muestran indispensables para la consolidación de la economía del conocimiento, además de contribuir al desarrollo económico, social y cultural de los países, y también para ayudar a los individuos a alcanzar las metas y retos deseables en el ámbito profesional, laboral y académico que la sociedad demanda como condición indispensable en la escala de valores requeridos actualmente, necesidad ineludible de nuestra sociedad global.

No obstante sabemos que existen diferencias notables en cuanto a la penetración, uso, acceso y habilidades de las TIC [Caridad, Ayuso y Ayuso, 2010: 56], la denominada brecha digital, que contribuye a reforzar las diferencias que se dan entre países, ha conformado una brecha tecnológica sin duda resultado de otras brechas sociales, económicas, políticas, generacionales, geográficas, culturales, etc. y, por supuesto, una también de género, objeto de la investigación que presentamos. La brecha digital es un problema social [Pimienta, 2008] y las diferencias de acceso entre hombres y mujeres en Internet reflejan una clara relación con los estudios, el nivel económico y la edad, como veremos cuando enfoquemos los términos de uso y las habilidades [INE y Ministerio de Igualdad, 2011]. La incorporación de las mujeres a la SI es un factor estratégico fundamental desde una perspectiva competitiva y de bienestar social, elementos clave para superar la actual situación económica, como nos recordó el European Institute for Gender Equality en su reunión de Vilnius (www.ec.europe/eu/dgs/justice/index_eu.htm).

Esta brecha que afecta en mayor o menor grado a todos los países, incluidos los más desarrollados, ha producido importantes exclusiones. La innovación y el desarrollo, sobre todo el avanzado, de las tecnologías no se difunden de forma regular debido a la inercia del propio sistema; de ahí la necesidad de establecer iniciativas que eviten la exclusión y potencien la inclusión digital de los ciudadanos [Castaño, 2008 y Castaño etal, 2010]. Sin embargo para propiciar las medidas necesarias que no permitan que esto suceda se impone conocer cúal es la situación específica acerca de la utilización real de las tecnologías. 
La literatura científica consultada, Korupp y Szydlik, [2005]; Brynin, [2006], entre otros, destaca los grandes beneficios de usar las TIC y sus efectos positivos, como se refleja, en las habilidades y competencias educativas, matemáticas, laborales, sociales, del lenguaje, etc. Se habla en efecto de un empoderamiento (empowerment). Compartimos la opinión de Castaño [2008: 23 y Castaño et al, 2010] de que la brecha digital es más compleja que el acceso a Internet porque existen más divisiones digitales. Desde esta perspectiva, como en otros muchos casos, las mujeres se presentan, grosso modo, como un factor catalizador de otras brechas sociales. Quizá iniciarse el siglo actual, las mujeres hayan percibido a las TC como un elemento de poca utilidad, pero hoy en día ya han reconocido la importancia no sólo del ordenador sino también de la tecnología móvil. Según la Fundación Orange [Informe, 2010], el caso de la utilización del móvil por parte de las mujeres o los envíos de "sms", e incluso el acceso a Internet por medio de la telefonía móvil, sobre todo en las mujeres jóvenes, se ha incrementado notablemente. De igual modo las mujeres se han incorporado al comercio electrónico o a la administración en línea en la medida que el sistema permite su inclusión a través del sistema educativo y que se han involucrado los poderes públicos, como veremos más adelante. Hoy, a pesar de que los hombres dominan los campos de la innovación, las mujeres se han incorporado al empleo cualificado, a los negocios y al ámbito de la investigación.

Ya hace años conocíamos por Roger [2001] que existe una brecha existe entre personas que se benefician de Internet y otras que están en desventaja a este respecto. El autor relaciona esto con la brecha del conocimiento; en tanto, que al aumentar el nivel socioeconómico, unas personas tienden a apropiarse de la información más rápidamente que otras de niveles sociales más bajos. Efectivamente, conforme aumenta la condición educativa, social y económica de la población, el desarrollo de las TIC no sólo es más rápido, sino que el nivel de habilidades es más avanzado o muy avanzado [Castaño etal, 2010]. Se generá así una brecha de conocimiento que, a nuestro juicio incidirá de manera sustancial en el acceso no solo a un empleo sino a uno cualificado, en una empresa, en la investigación, en la alta dirección, etc. Esta brecha de conocimiento generada por la brecha digital es la que más afecta a las mujeres, entre otras cosas por la rapidez que lleva la evolución tecnológica y la incorporación continua de habilidades y formas de conducta que propicia nuevas, ya que si bien en algunos casos las mujeres han modificado sus costumbres y producido cambios cualitativos y cuantitativos, en otros, han reforzado los estereotipos de género que las relegan en el mercado de trabajo [INE y Ministerio de Igualdad, 2011]. Si somos conscientes de que la brecha digital de género aumenta las diferencias profesionales y laborales, veremos 
que en general éstas se traducen en términos salariales, estabilidad laboral, prestigio profesional, etc., y que provocan una mayor desigualdad al sumarse a los ya existentes roles producidos tradicionalmente: ser madres, amas de casa, empleadas y trabajadoras de nivel bajo, lo que produce situaciones precarias económicamente debido a esta dedicación familiar, que las hace dependientse económica y socialmente.

Por otro lado, una mayor utilización de las TIC podría incentivar a que las mujeres tomaran una parte activa en foros y redes, en la defensa de sus propios derechos, en el acceso a nuevos y mejores trabajos de calidad, e incrementaran sus oportunidades para recibir información cualificada no disponible en otros medios convencionales, y si en bibliotecas digitales, bases de datos, repositorios institucionales y en general todo el acervo de conocimientos accesible en Internet.

Establecido el problema, la presente investigación se propone, en primer lugar mostrar cuál es la situación real de la brecha digital de género en España y compararla con la de otros países de la UE. En segundo lugar, se centran en establecer cuáles son las medidas más adecuadas para corregir esta tendencia, conscientes de que sin contar con datos concretos; y mediciones no hay visibilidad y sin ésta no hay credibilidad, según Hafkin [2006]. Por tanto a partir del análisis de los datos se revisan las medidas pertinentes de inclusión social que serian necesarias para paliar la exclusión de las mujeres de las TIC. Así, se focaliza el problema, se presentan datos estadísticos sobre la utilización de las TIC, con indicaciones de género en España y en las Comunidades Autónomas, esto es en los gobiernos regionales, en el contexto de la Unión Europea, y se indican medidas de mejora para alcanzar una efectiva cohesión social.

Según E. Ortoll Espinet [2007] las TIC en sí mismas no garantizan la inclusión social, sino que se debe buscar su adecuación a las necesidades de cada colectivo. Por otra parte, la OCDE [2006] ya estableció tres categorías según la excelencia de uso por parte de los sectores de usuarios, a) básico (utilizan Word, ppt, Excel; b) avanzados (herramientas de software; y c) especialistas (capacidad de crear y mantener sistemas). Es necesario tomar medidas de inclusión que incrementen no sólo el uso básico, sino también el avanzado y el muy avanzado de las TIC por parte de las mujeres en términos cuantitativos y cualitativos; se trata de disminuir la brecha, tanto de género en info-habilidades como en habilidades avanzadas y muy avanzadas.

Por último se revisan aquí las normativas de carácter general horizontal ya acometidas por los poderes públicos para evitar la exclusión, así como las medidas transversales y específicas que se están llevando a cabo en el ámbito de los gobiernos regionales. sobre inclusión digital para mujeres: Se señalan 
cuáles de estas medidas serían las más benéficas y se ajustarían mejor a las necesidades que demanda la sociedad española actual.

Hemos constatado que es necesario profundizar, a través de la investigación, tanto los factores que inciden en la inclusión digital de las mujeres como la problemática que implicaría lograr esto y cualificar su uso: usuarios básicos, usuarios avanzados y especialistas TIC, si bien no es éste el tema fundamental de este trabajo. Posteriormente Van Welsum y Montaigner [2007] establecieron categorías relacionadas con las mujeres para ayudar a romper este "techo de cristal" que parece no sólo impedir el desarrollo de las TIC por parte de las mujeres, sino aumentar la tasa de fractura digital en el conjunto del Estado Español. Como veremos la brecha digital de género es más profunda en las categorías de usuarios avanzados que utilizan con destreza herramientas de software [INE y Ministerio de Igualdad, 2011, Fundación Orange, 2011, Castaño etal. 2010 etc.], aunque las TIC no son la tarea fundamental de su trabajo, ni la de otros usuarios especialistas de la tercera categoría que se encuentran en la dirección, creación y gestión de TIC en los puestos más altos.

\section{Material y método}

Establecido brevemente el contexto del problema objeto de análisis, la brecha digital de género y el análisis de las medidas específicas de inclusión digital establecidas por los poderes públicos nacionales y autonómicos, puede apreciarse que se trata de un tema de interés y actualidad, que no sólo afecta a la presencia o ausencia de las mujeres en la SI, sino además a las desigualdades entre hombres y mujeres, y que tienen que ver con el desarrollo económico y la cohesión social de España en el contexto comunitario.

La hipótesis de partida que se ha establecido es si

la falta de incorporación de las mujeres a la SI, esto es, la brecha digital de género supone un obstáculo para alcanzar las metas propuestas en relación a la SI y poder corregir la actual brecha digital de España convergir con los países más industrializados de la UE,

con lo cual nos referimos a la UE de los 15, y ampliamos la hipótesis para reflexionar si las medidas de inclusión digital que se establecen en nuestro país son adecuadas y pertinentes para cerrar la brecha digital.

Los materiales utilizados para la realización del presente artículo son la literatura científica citada y una gran diversidad de fuentes: Diario Oficial de la Unión Europea (DOUE), Boletín Oficial del Estado (BOE) informes de la 
OCDE de la Fundación Orange, Unión Internacional de Telecomunicaciones, Instituto Nacional de Estadística, INE de España etc., y otros recursos relacionados con los objetivos planteados por las autoras, tanto normativos, como indicadores de la UE [Eurostat, 2010], y otros organismos e instituciones internacionales [Connectivity Scorecard,2010], además de los datos suministrados por las Comunidades Autónomas, no siempre de fácil acceso.

El ámbito de estudio es España por lo que conviene tener en cuenta su estructura en Comunidades Autónomas; es decir, los gobiernos regionales que tienen competencias para realizar acciones y planes específicos en su ámbito.

Este estudio se realizó del 2003 al 2010.

La metodología propuesta para alcanzar los objetivos marcados es la propia de las Ciencias Sociales y Humanas, en general y de las Ciencias de la Documentación en particular.

Se tomó en cuenta la normativa vigente sobre la Sociedad de la Información en la UE y en España y se analizaron los datos estadísticos a través de su análisis comparativo en el contexto comunitario. En primer lugar se estableció la evolución porcentual sobre la utilización de Internet por parte de los ciudadanos (2003-2010) en la Europa comunitaria de los 15; esto es, los países europeos más industrializados, y la actual UE constituida por 27 estados actualmente. Posteriormente se realizó un análisis cuantitativo con indicación de género. Se presentan además los datos segregados por sexo en las CCAA, conscientes de que la medición cuantitativa documenta los datos reales sobre la cuestión pero no refleja las causas cualitativas, ni tampoco toda la realidad sobre la brecha digital de género, dado que los indicadores que contemplan la medición son poco específicos y no contemplan la complejidad y profundidad de la brecha en toda su amplitud, como tampoco lo hacen los indicadores sintéticos establecidos por la UE. Prueba de ello es que ya existe en la agenda comunitaria la necesidad de revisar tales indicadores, como se vio en la Reunión de la Comisión Europea en Granada (Europa 2010) en la que se contempló la necesidad de hacer mejoras en este rubro. También en nuestro país la "ley Orgánica 3/2007 para la igualdad efectiva de hombres y mujeres" sostiene en su articulo 20, apartado b) que se deben

Establecer e incluir en las operaciones estadísticas nuevos indicadores que posibiliten un mejor conocimiento de las diferencias de los valores, roles, situaciones, condiciones, aspiraciones y necesidades de mujeres y hombres, su manifestación e interpretación en la realidad que se vaya a analizar.

Como se aprecia se alude a la necesidad de adecuar de los indicadores en tiempo y forma al considerarlos cambiantes y adecuados a la evolución social. 
Finalmente se analizan los planes de inclusión digital en las CCAA y se presentan conclusiones.

\section{Contexto de igualdad digital de GÉNero: MEDIDAS NORMATIVAS}

A continuación se analizan las medidas normativas de carácter general y horizontal de la e-igualdad en la UE y en España, así como los planes de inclusión en el conjunto del Estado, ya que afectan al desarrollo de la Sociedad de la Información en su conjunto.

\section{A) En la Unión Europea:}

Apenas se recuerdan las primeras acciones de creación de la Sociedad de la Información en la Cumbre de Lisboa en 1999 y las posteriores iniciativas eEurope 2000, e-Europe 2002, e- Europe 2005 y la cercana 2010 [2007], con la que se inicia el ciclo sobre la inclusión social para ser parte de la Sociedad de la Información para todos.

Está en desarrollo la actual Comunicación de la Comisión Europea, Acción Normativa, 2020, "Europa 2020, Una Estrategia para un Crecimiento Inteligente, Sostenible e Integrador" [2010] nacida con la vocación de evitar una segunda brecha digital más peligrosa y difícil, "la brecha de conocimiento". Esta comisión contextualiza las TIC en su conjunto en el entorno de la Investigación, el Desarrollo tecnológico y la Innovación $(\mathrm{I}+\mathrm{D}+\mathrm{I})$, de una forma gráfica, comprensible y forma parte de un todo. Esto quiere decir que antes la 2010 no formara parte de una planificación unitaria integradora como la actual 2020.

Ahora la estructuración depende de tres ejes de crecimiento: uno "inteligente" basado en el conocimiento y la innovación; uno "sostenible", que implica una economía competitiva y sostenible, y uno "integrador" para promover y conseguir un empleo duradero y luchar contra la pobreza. En cuanto a las medidas de inclusión digital, la estrategia 2020 establece objetivos concretos y precisos en materia de rendimiento que se alcanzarán en 2015 y buscarían la inclusión digital de los ciudadanos, en general, y en particular la de ciertos sectores desfavorecidos. Elementos destacados son:

- Un aumento de la utilización de Internet en la población buscando alcanzar de un $60 \%$ a un $75 \%$ de uso regular .

- Se pretende para antes del 2015, pasar del actual 41\% al 60\% de participación en colectivos desfavorecidos. 
- Se quiere disminuir a la mitad la población que nunca ha utilizado Internet: hasta un $15 \%$ y un $30 \%$ de personas comprendidas entre los 16 y los 74 años.

La "Agenda Digital para Europa". Para completar estas medidas se establece una iniciativa emblemática propuesta en esta ocasión por la Presidencia Española de la Comisión Europea, la "Agenda Digital para Europa" (2010), con la finalidad de conseguir beneficios económicos y sociales sostenibles a través de:

- Un Mercado único digital basado en un acceso a Internet rápido y ultrarrápido, y ciertas aplicaciones ínteroperativas para todos para el 2013.

- La creación de un mercado único de contenidos y servicios en línea seguro y sin fronteras.

- Reforzar los fondos destinados a la I+D+I en el campo de las TIC,

- Promover el acceso y la utilización de Internet para todos los ciudadanos europeos mediante actividades que apoyen la alfabetización digital y la accesibilidad.

\section{B) España}

En los últimos años existen también en España medidas de carácter general y horizontal que van encaminadas a establecer espacios de igualdad social entre los ciudadanos. Señalamos a continuación algunas de las medidas más destacadas en este sentido, que han sido adoptadas en campos estratégicos y que suponen un punto de partida para evitar la exclusión social en general y la de género en particular:

- En el ámbito de la Educación, La Ley 2/2006, LOE (Ley Orgánica de Educación) establece una educación obligatoria y gratuita para todos los ciudadanos hasta los 16 años.

- El Plan Nacional de Acción para la Inclusión Social del Reino de España 2008-2010.

- La Ley Orgánica 3/2007 (Ley de Igualdad), para la igualdad efectiva entre hombres y mujeres, y el posterior Plan de Igualdad de Oportunidades entre Hombres y Mujeres (2008-2011), refuerzan y complementan de alguna manera la normativa existente a la vez que corrigen desviaciones de uso y acceso a las TIC y otros servicios, debidas a los desajustes provocados en el entorno económico y social de los ciudadanos. 
Otras acciones de los Ministerios de Industria, Turismo, Comercio y el de Ciencia e Innovación, también han adoptado medidas en este sentido. Destacamos el Plan Red.es (2000) www.red.es Redes es la Entidad Pública Empresarial adscrita al Ministerio de Industria destinado a las pequeñas y medianas empresas (PYMES), y el Programa Ingenio 2010 [2005] cuyos instrumentos son los Programas Cenit, Consolider (no desarrollados por problemas presupuestarios) y el Plan Avanza 2; sólo éste último funciona satisfactoriamente a través de la "Estrategia 2011-2015, Medidas de Impulso a la S.I". Esto último, como argumentamos en el inicio de esta investigación, está ligado al Ministerio de Ciencia e Innovación de modo que, la normativa referente a las ayudas a TIC se incorporan al Plan de I+D +i (2008-2011) nacional.

Sin embargo a pesar de estas medidas se producen equilibrios y desequilibrios en la asunción de ellas, por lo que no todos los sectores sociales se ven afectados de la misma forma. En Primaria y Secundaria las desigualdades entre chicos y chicas en cuanto a info-habilidades en TIC no tienen sesgo de género, según datos del Ministerio de Educación [INE, 2011] y sólo existe una leve diferencia entre los chicos que representa un $93,6 \%$ y las chicas un $91,2 \%$, y sí existe cierta brecha en algún sector, pero ésta está determinada por otras brechas sociales a las que el sistema no alcanza.

En la Universidad incluso mejora esta situación. Con más de un 50 \% de mujeres jóvenes, éstas compiten en destreza y habilidades TIC, con resultados mejores, en la mayoría de los casos, con respecto a sus compañeros [INE, 2011]. Así que la brecha digital de género sólo es indicativa del resultado de comportamientos "comunes" y de estereotipos y roles difícil de erradicar totalmente en la sociedad. Esta brecha digital de género, sin embargo, aumenta cuando se va escalando hacia los niveles educativos más altos. En estudios superiores relacionados con la Arquitectura y las Ingenierías el porcentaje de alumnado femenino decrece sustancialmente en Arquitectura 31,15€ y en Ingenierías en un 24,6\%. Hay que tener en cuenta que la Estadística de la Enseñanza Universitaria en España cifra en un 54,3 \% las alumnas matriculadas en primero y segundo Ciclos. El número de varones doctores, sin embargo, es superior al de las mujeres en todos los tramos de edad 10,6\%, excepto entre los y las menores de 35 años, que es un punto superior 11,6\% para las mujeres [Fundación Orange, 2011; INE, 2011].

Esta brecha de género se repite también en los niveles profesionales de la gestión de empresas: industria, innovación, alta gestión, dirección, etc., tal como lo refleja el Gráfico 1 del Instituto Nacional de Estadística, según 
el Ibex 35 , "Índice oficial de referencia de la Bolsa española elaborado por Bolsa y Mercado", donde aparece que solo un $2.9 \%$ de las mujeres ocupan puestos como presidentas de grandes empresas que cotizan en los mercados, y un poco más un 7.8 \% son vicepresidentas. La representación más alta corresponde a su participación como Consejeras y como Secretarias: un $11.1 \%$ y un $14.3 \%$, respectivamente, [INE y $\mathrm{M}^{\circ}$ Igualdad, 2011].

Gráfico 1. Mujeres en Consejos de Administración Ibex 35 - 2009

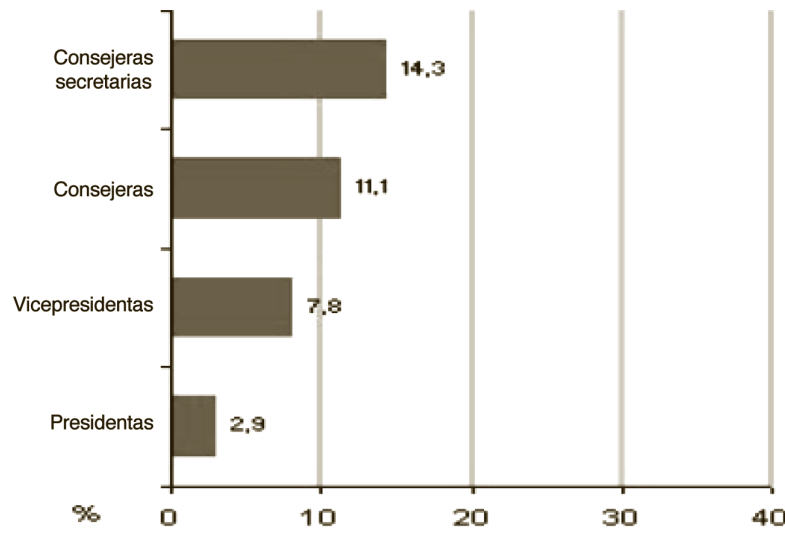

Fuente: INE a partir de datos de la Comisión Nacional del Mercado de valores

Así pues, parece también necesario adoptar medidas de e-igualdad de género que propicien la inclusión social en general y la inclusión digital de género en particular, para poder conseguir mayores habilidades TIC que propicien competencias en investigación y tecnologías para alcanzar una mejora laboral y un mejor salario en los niveles altos de las empresas, además de profundizar en la consecución de info-habilidades entre las mujeres para alcanzar una mayor cohesión social.

\section{Resultados y DiscusióN}

Según la literatura científica revisada se puede afirmar que, efectivamente, existe la brecha digital de género en distintos niveles de la sociedad y que se deben tomar medidas de inclusión digital en España para mejorar esta situación. Lo primero que deseamos resaltar es que escasean las fuentes y los estudios especializados que traten en profundidad la problemática abordada en este trabajo de investigación. Ahora bien, somos conscientes de que la realidad puede desdibujarse y de que no sólo el análisis cuantitativo sea necesario 
para diagnosticar el problema, de ahí que la fundamentación teórica sea de interés, junto con otras fuentes institucionales públicas y privadas, aunque estas últimas no incidan en la necesidad de tomar medidas de inclusión para conseguir una cohesión social efectiva.

A continuación se presenta, la situación real de los datos cuantitativos en el contexto comunitario sobre la situación de la SI en España.

En el Gráfico 2, tal y como indicamos en la metodología, se muestran datos de carácter general que recogen la evolución del porcentaje de particulares; esto es, tanto hombres como mujeres (16-74 años) que utilizaron regularmente Internet en el período 2003-2010, para posteriormente establecer una comparación entre hombres y mujeres.

Gráfico 2. Evolución porcentaje de utilización de Internet de particulares.

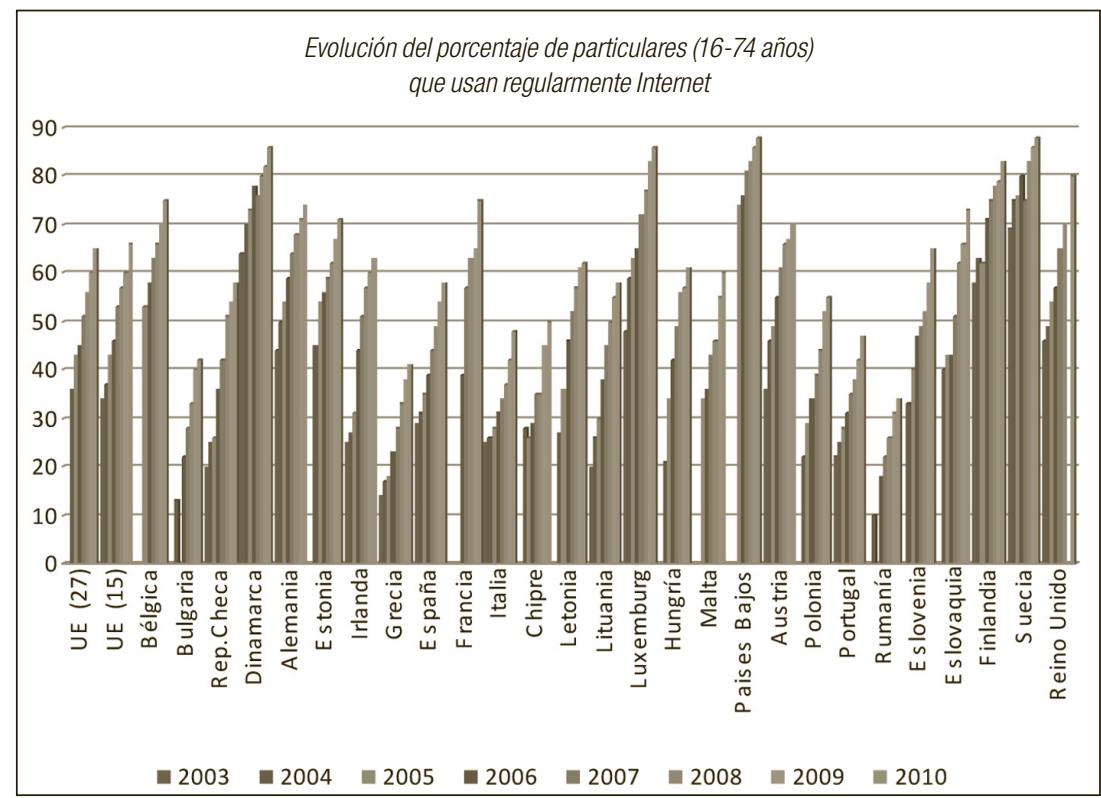

Fuente: Eurostat y elaboración propia

El primer análisis comparativo se establece entre el porcentaje de particulares (de 16 a 74 años) que usan regularmente Internet, en la UE (15), UE (27) y España. En una primera lectura del Gráfico 2, cuyos datos aparecen en la tabla 1, se percibe que el punto de partida, $37 \%$, de utilización en 2004 para la UE (15) y un $31 \%$ para España, presenta un desfase de 6 puntos de desventaja de nuestro país respecto a los países más industrializados de Europa. Si la comparación se hace respecto de la UE (27), el desfase en ese año es de 5 puntos. La evolución de la situación en España de 2004 a 2010 nos da 
un incremento de 27 puntos, mientras que la evolución en UE (15) y UE (27) fue de 29 puntos en ambos casos. De ello se puede deducir que la brecha general de partida no sólo se mantuvo sino que aumentó ligeramente y parece haber crecido ligeramente al término del periodo analizado. Esto pone de manifiesto el crecimiento de España [INE, 2010, 2011], pero sobre todo que éste debe aumentar y ser sostenible a pesar de lo controvertido de nuestros datos económicos actuales.

Tabla 1: Evolución del porcentaje de particulares (16-74 años) que usan regularmente Internet.

$\begin{array}{lcccccccc}\text { Particulares } & 2003 & 2004 & 2005 & 2006 & 2007 & 2008 & 2009 & 2010 \\ \text { UE (27) } & & 36 & 43 & 45 & 51 & 56 & 60 & 65 \\ \text { UE (15) } & 34 & 37 & 43 & 46 & 53 & 57 & 60 & 66 \\ \text { España } & 29 & 31 & 35 & 39 & 44 & 49 & 54 & 58\end{array}$

Fuente: Elaboración propia

Establecido el marco de la brecha digital en España se estudia la situación de los hombres en el mismo periodo y lugares, y se señalan con claridad algunos aspectos que este tipo de indicadores presentan (Se han contrastado indicadores de la Fundación Orange de la Unión Internacional de Telecomunicaciones. El INE, 2010 y 2011, señala datos semejantes; no olvidemos que la fuente de Eurostat es El Instituto Nacional de Estadística español.

A continuación (Gráfico 3) se analizan los que existendatos sobre la utilización regular de Internet por hombres, los cuales ratifican la tendencia de la tasa al alza. Si los estudiamos con atención, también, demuestran la brecha existente entre España y los otros países del entorno comunitario, además de la brecha de género que veremos a continuación detenidamente. 
Gráfico 3. Evolución porcentual de hombres que usan Internet

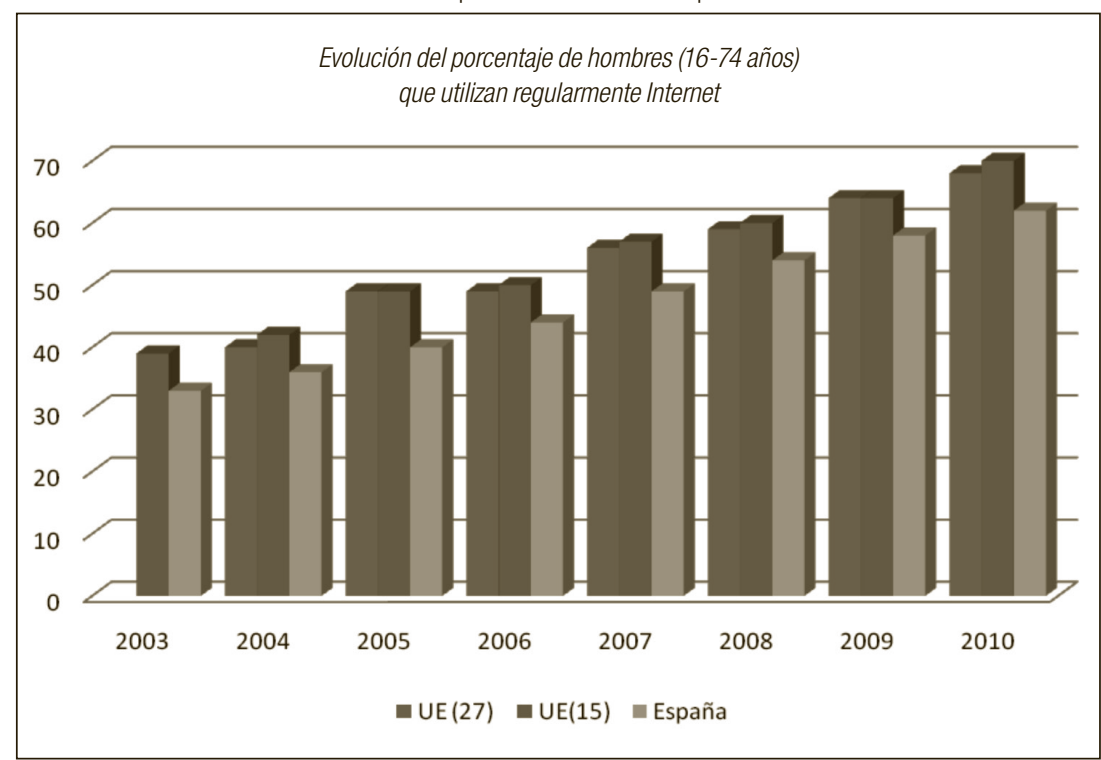

Fuente: Eurostat y elaboración propia

Se muestra a continuación el (Gráfico 4, Tabla 2) con la evolución del porcentaje de mujeres que utilizan regularmente Internet. Como se observa, la diferencia entre hombres y mujeres, según los datos anteriores, difiere sustancialmente, y como afirma Castaño et al [2010] esta brecha es difícil de modificar porque hay que añadirle otras que son educativas, sociales, culturales y de nivel económico, todo lo cual refuerza los estereotipos que obstaculizan la incorporación plena de las mujeres a la SI. Este aspecto se destaca más todavia en una época de incertidumbre económica como la actual . 
Gráfico 4. Evolución porcentual de mujeres que usan Internet

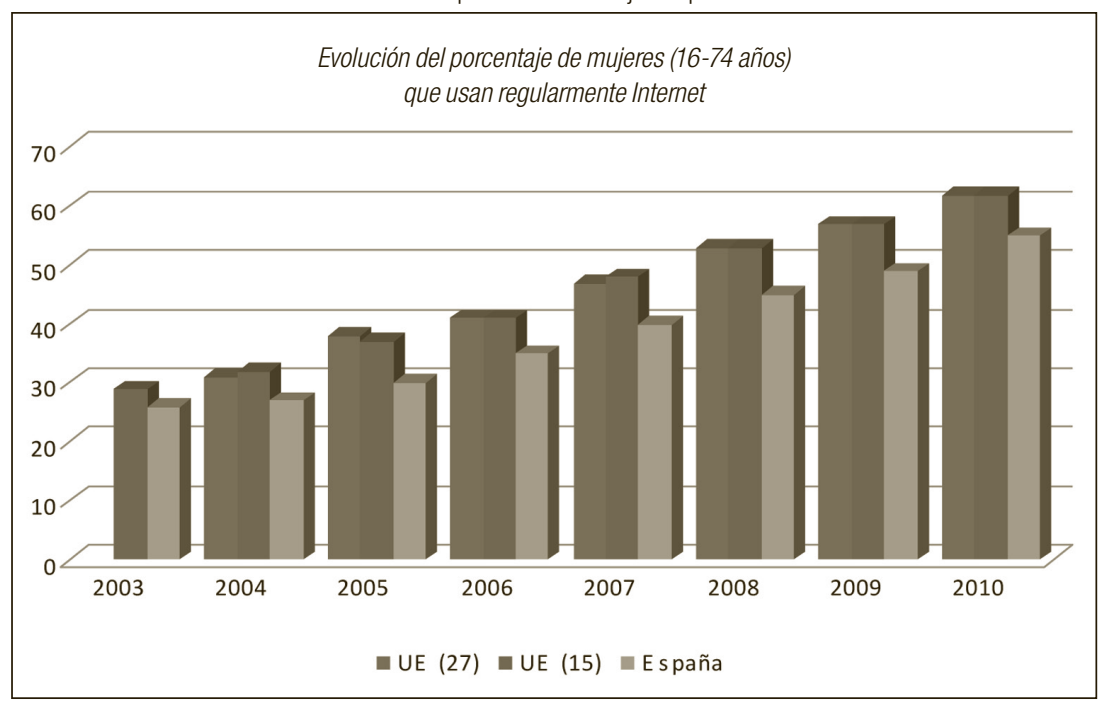

Fuente: Eurostat y elaboración propia

Desde la primera lectura de los Gráficos (3 y 4) se aprecia que la brecha digital de género en España no es homogénea, aunque datos muy recientes del INE [2011] apuntan a una cierta disminución de la homogeneización regional. La tasa de utilización de Internet por mujeres apenas se reduce en el periodo estudiado. Y con un análisis comparativo se aprecia que la brecha digital de género en España, que se mantenía en los 9 puntos desde 2004 a 2009, pasa a 7 puntos en 2010; es decir experimenta en el último año estudiado una ligera mejoría, como se observa en la Tabla1. Sin duda esta mejora tiene una relación directa con las medidas de inclusión emprendidas por diversos colectivos de mujeres, sobre todo aquellos que se encuentran en la pirámide generacional de entre los 45 a los 74 años, que es cuando se produce una brecha mayor, [INE y $\mathrm{M}^{\mathrm{o}}$ de Igualdad, 2011].

Comparando la situación de nuestro país respecto a Europa, en cuanto a la utilización de Internet por parte de los hombres españoles respecto a los europeos de la UE (27 países miembros de la UE), la brecha oscila entre los 4 y los 9 puntos del 2004 al 2008 y se estabiliza en 6 puntos en los dos últimos años estudiados (2009 y 2010); es decir, se produce una disminución estable. En lo referente a las mujeres españolas respecto de sus contrapartes de la UE (27), la oscilación se produce entre los 4 y los 8 puntos desde 2003 a 2008, y disminuye levemente a 7 puntos en 2010, como se puede apreciar en la Tabla 2 . 
Tabla 2: Evolución del porcentaje de hombres y mujeres (16-74 años) que utilizan regularmente Internet en España.

$\begin{array}{lcccccccc} & 2003 & 2004 & 2005 & 2006 & 2007 & 2008 & 2009 & 2010 \\ \text { España Hombres } & 33 & 36 & 40 & 44 & 49 & 54 & 58 & 62 \\ \text { España Mujeres } & 26 & 27 & 30 & 35 & 40 & 45 & 49 & 55 \\ \text { UE (27) Hombres } & & 40 & 49 & 49 & 56 & 59 & 64 & 68 \\ \text { UE (27) Mujeres } & & 31 & 38 & 41 & 47 & 53 & 57 & 62\end{array}$

Fuente: Eurostat y elaboración propia

Estos datos nos permiten valorar que no hay una eliminación significativa de la brecha de género en España y además que no hay convergencia con la Europa comunitaria (27). Aunque la comparación con europa muestra en ambos casos una inflexión positiva en el 2010, ya muestra una tendencia al estancamiento según los datos del INE del 2011. Esta debilidad de la posición española puede deberse a la disminución de los fondos económicos destinados a la ejecución del Plan Avanza2, [PLAN AVANZA2, 2010]. En lo que concierne con la brecha de género en la utilización de Internet en la UE (27), tal y como se deduce de los datos aportados, esta investigación muestra que no es sino hasta los tres últimos años cuando se percibe una disminución que alcanza los 6 puntos de diferencia en el 2010 (inferior a la brecha de género en España).

De lo anterior concluimos que aunque las medidas adoptadas por los poderes públicos tanto comunitarios (2010, 2020, Agenda digital para Europa etc.) como españoles (Programa Ingenio, Plan Redes, Pan Avanza2 etc.) han ejercido un ligero efecto positivo que aprecia en el 2010, aunque no ha eliminano la brecha de género, que se mantiene tanto en la UE (27) como en España. La agenda Digital para Europa [2010] en su "pilar 6" propone ciertas medidas para que las TIC puedan ser adoptadas por parte de los grupos excluidos.

Por otra parte en la representación en líneas (Gráfico 5), datos ya comentados, puede apreciarse más gráficamente que no existen diferencias notables en la tasa de utilización de Internet por particulares entre la UE de los quince países más industrializados y la UE de los veintisiete países miembros que la conforman en la actualidad. 
Gráfico 5. Evolución del porcentaje de particulares que usan Internet

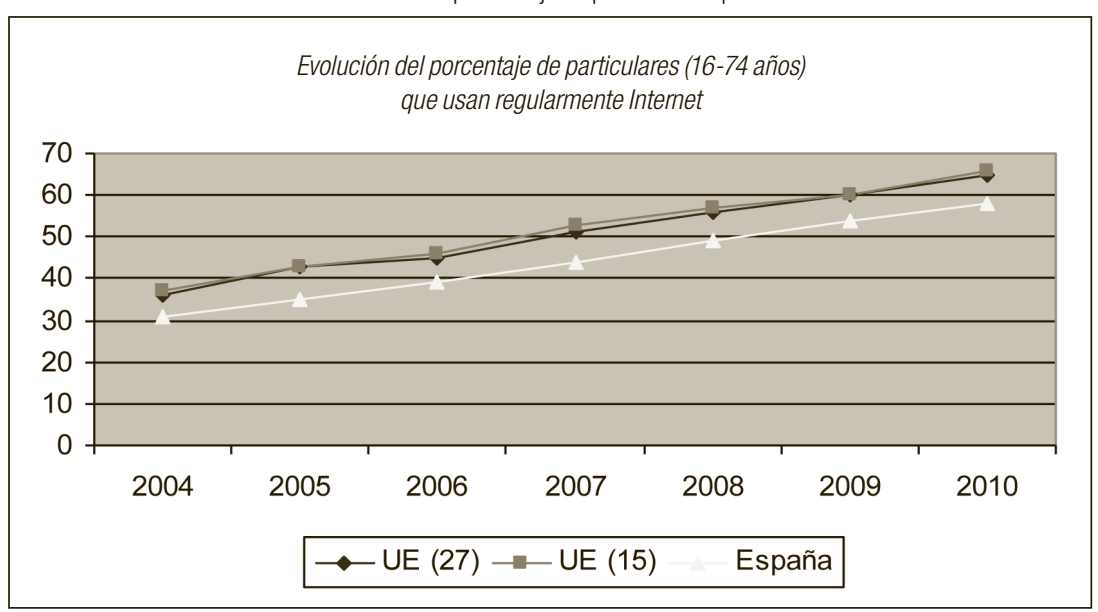

Fuente: Eurostat y elaboración propia

Como se observa la incorporación, de nuevos países a la UE en 2004: Polonia, Hungría, Republica Checa, Lituania, Estonia, Letonia, Eslovaquia, Eslovenia, Malta, Chipre, más la incorporación de Rumania y Bulgaria en 2006, no ha supuesto merma en la tendencia del crecimiento sostenido, aunque sí se manifiesta una fractura respecto a España.

Con respecto al equipamiento y uso de las tecnologías, presentamos a continuación una representación (Gráfico 6) del año 2010 por grupos de edad en España. Como ya comentamos en la fundamentación de este trabajo, la exclusión digital se agrega a otras brechas, entre las que se encuentran las padecidas por personas de entre 45 a 74 años. Para paliar esta situación una parte importante de la Agenda Digital para Europa [2010] está enfocada a paliar el envejecimiento población con ayuda de las TIC y a ayudar a ésta a obtener una mejor calidad de vida. Este objetivo tiene como fecha para alcanzarse en el 2015. 
Gráfico 6. Equipamiento y uso de tecnologías de la SI en hogares españoles

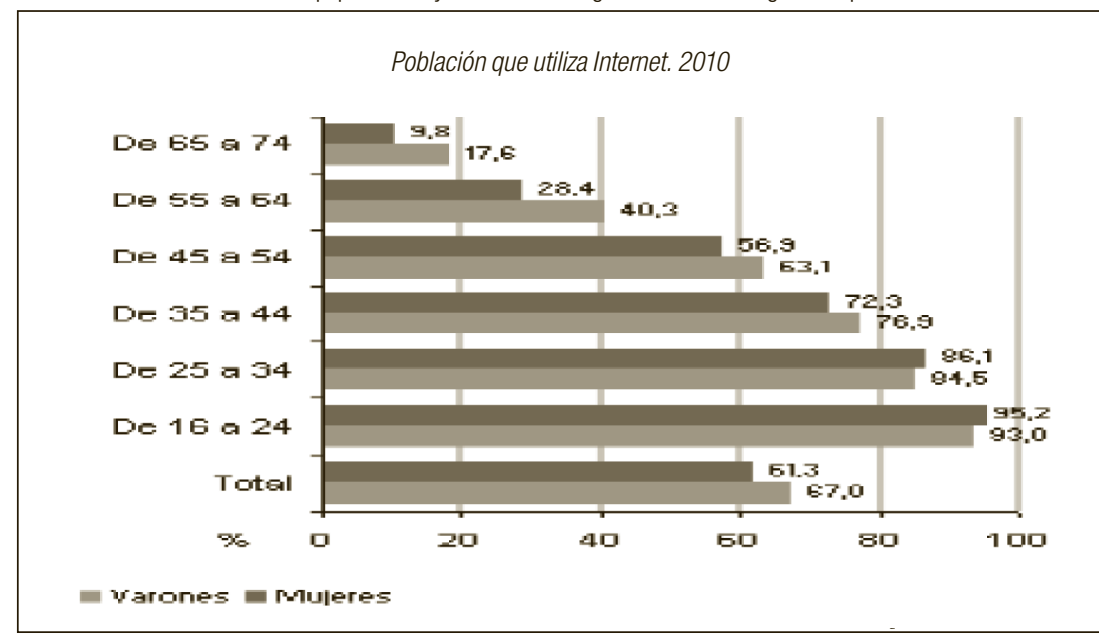

Fuente: Encuesta sobre equipamiento y uso de Tecnologías de la Información y Comunicación en los hogares. INE

Este gráfico completa los datos anteriores y pone de manifiesto el argumento defendido al comienzo de la investigación, de que entre los jóvenes de entre 16 a 24 años [INE, 2011] apenas existe apenas brecha digital de género ( 2 puntos), situación que se prolonga hasta el intervalo de edad de 25 a 34 años. En el citado gráfico en este grupo de edad ya decrece la utilización de las TIC y llega casi a 10 puntos la brecha de género entre las mujeres de 16 a 44 años. Es en los umbrales de este grupo de edad cuando comienza hacerse más profunda la brecha digital de género que es una de las debilidades que tanto los poderes de la UE como el español deben tener muy en cuenta al establecer acciones y medidas normativas nuevas.

Por último cabe recordar que la inclusión digital está estrechamente ligada a las políticas de la UE en materia de inclusión social, la educación y la cultura, el desarrollo regional, la innovación, la industria, y el mercado interior [2020].

\section{Situación en las Comunidades Autónomas (CCAA)}

Conviene aclarar que los gobiernos regionales en España, tienen ciertas competencias que les permiten realizar acciones en sus regiones para incentivar la Sociedad de la Información y otras medidas de inclusión entre sus ciudadanos. De ahí el interés de este epígrafe. En las Comunidades Autónomas se establecen comparativas de la brecha digital por sexo, teniendo como referente la media nacional. Tal y como lo reflejan los datos más recientes, la tasa 
de la brecha digital de género no es homogénea y difiere según las regiones, (Gráfico, 7) y los planes de acción llevados a cabo, (Anexo 1) si bien los datos más recientes, como hemos indicado anteriormente, pronostican una homogeneización en los entes regionales debido a las acciones sobre la SI y medidas de inclusión orientadas hacia los colectivos excluidos (Anexo 1)

Gráfico 7. Personas que han utilizado Internet en la CCAA

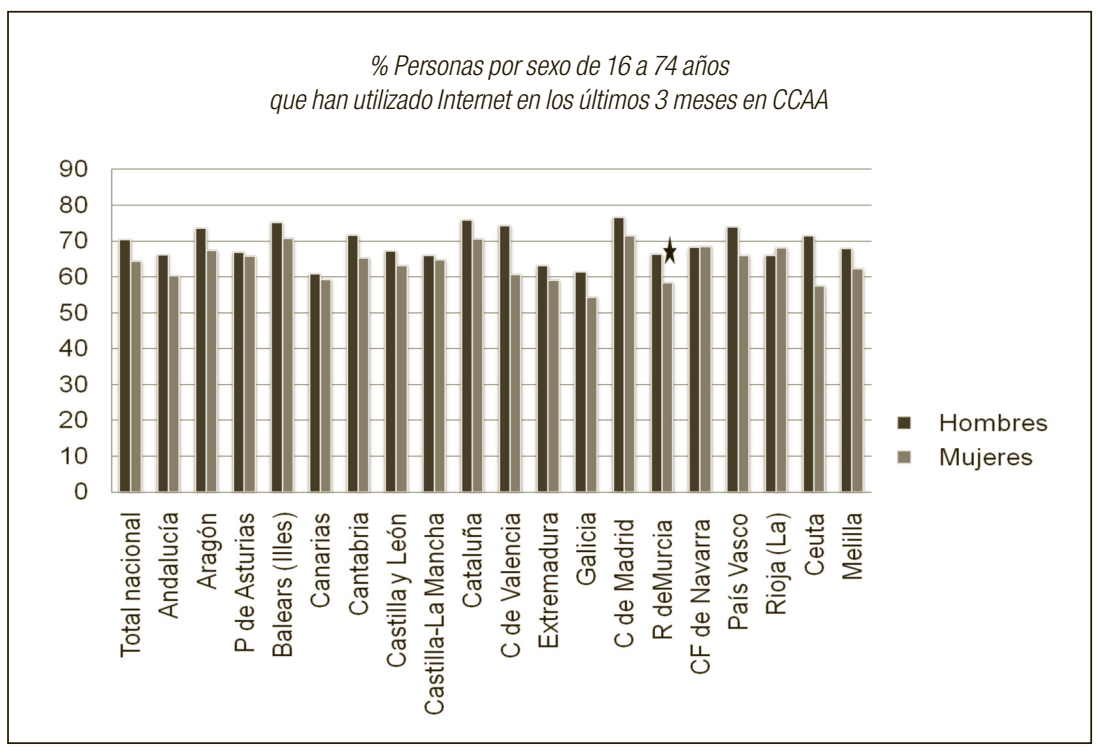

Fuente: Elaboración propia a partir de datos INE

Se aprecia aquí que en las Comunidades Autónomas más ricas, esto es aquellas que tienen un PIB mayor [NE, 2010, INE, 2011] la brecha digital es menor y supera la media nacional de acceso a las TIC al convergir con los países más desarrollados de la UE. Es el caso de Madrid, Cataluña y las Islas Baleares, que superan los 70 puntos porcentuales, pero también otras regiones como Asturias y Aragón están muy cercanas a estos parámetros.

Por otra parte también se observa que no siempre se refleja una mejora de la participación de las mujeres en la SI. Si analizamos los valores de la brecha de género vemos que existe un estándar semejante en las CCAA más allá de los datos porcentuales generales. Es decir que en las regiones mencionadas disminuye la brecha digital en su conjunto, pero no la que existe entre hombres y mujeres [Castaño et al. 2010]. Se establece de este modo, como decíamos, un "techo de cristal" sólo superable si se toman las medidas adecuadas de inclusión digital de género adoptadas por los poderes públicos nacionales y regionales. 
En este sentido la investigación ha llegado a los resultados siguientes teniendo en cuenta los indicadores que se detallan: $1^{\circ}$ qué CCAA tenían programas específicos de inclusión y $2^{\circ}$, qué CCAA tenían planes avanzados, ( Figura 1 y Anexo 1).

Fig. 1. CCAA que tienen planes específicos y nivel de ellos
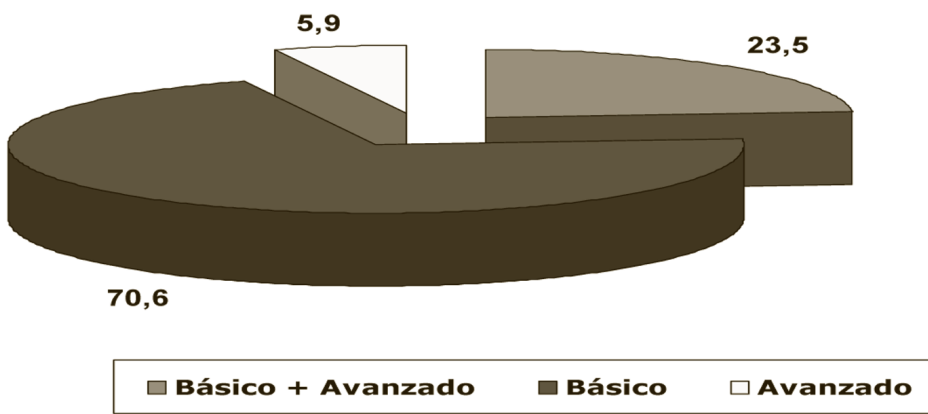

Fuente: elaboración propia a partir de datos de las CCAA

Como se puede deducir, un alto porcentaje de Comunidades autónomas (el 70,6\%) disponen de planes básicos, dirigidos a colectivos de asociaciones, personas mayores, empleados etc.; mientras que sólo un 5,9 \% dispone de planes de nivel avanzado, aunque un 23,5\% de ellas cuenta con planes específicos de ambos niveles. Sin embargo ninguna comunidad contempla cursos, planes o acciones dirigidas a conseguir habilidades avanzadas ahí donde la brecha es mayor, [INE, 2011; Castaño et al 2010]; se estima, sin duda, que esta alfabetización debe ser algo personal e individual.

Hay que decir, que la ausencia de seguimiento y sistemas de evaluación relacionados con el cumplimiento de los citados planes y sobre las personas a las que atiende; es decir los usuarios efectivos de esos planes y el grado de satisfacción obtenido por la población en relación con las medidas de inclusión adoptadas, impide poder extraer consecuencias o conclusiones específicas por parte de las CCAA, salvo las ya expuestas, dado que no ha sido posible acceder a informes de este tipo, suponiendo que existan.

\section{Conclusiones}

Los datos obtenidos de la investigación ponen de manifiesto la hipótesis de partida que establece que la brecha digital de género supone un obstáculo para alcanzar las metas propuestas en relación a la Sociedad de la Información en España y poder converger éstas con las de la UE. De ello pueden 
deducirse dos hechos: primero que las medidas adoptadas son insuficientes tanto en lo que afecta a las mujeres de la UE, en menor grado, como a las españolas, y que el problema atañe al propio colectivo de las mujeres en su conjunto, por lo que éstas tendrán que aplicar medidas más selectivas y transversales, no sólo en lo que se relaciona con las info-habilidades sino, sobre todo en lo que tiene que ver con crear sinergias que propicien competencias y habilidades avanzadas y muy avanzadas; esto es, meta-habilidades para las mujeres. El segundo hecho a tener en cuenta es que las medidas ya adoptadas, como la Ley de Igualdad y el Plan de acción para la igualdad real entre hombres y mujeres, y otras acciones pertinentes, son un caso de actuación integral y global sobre las distintas fracturas de nuestra sociedad y que habrá que esperar a ver los resultados y comprobar si estas actuaciones pueden romper ese "techo de cristal" que parece impedirles a las mujeres un despegue mayor en la SI y hacerlas más visibles en la sociedad y en la toma de decisiones del país,

El contexto mundial, globalizado y cambiante en el que vivimos requiere no sólo habilidades básicas, sino también avanzadas y muy avanzadas o especializadas en TIC para poder afrontar los retos actuales y evitar la Brecha Digital, además de desarrollar también competencias y habilidades "meta", para evitar la brecha de Conocimiento, en general por parte de toda la ciudadanía y de forma específica a los grupo "meta", muy cualificados, [Castaño et al, 2010], del colectivo de mujeres,

Además hay que tener en cuenta que la accesibilidad y el uso de Internet no sólo son una desventaja cuantitativa, sino que predetermina una ya presente y futura brecha de conocimiento. Se debe potenciar la utilización de indicadores cualitativos y sintéticos adecuados tendientes a controlar esas desviaciones, y propiciar que los datos sean un reflejo real de la situación existente. Por ejemplo se deben intensificar los indicadores, no sólo sobre el uso de Internet, sino también ponderar las variables de éstos que nos permitan establecer con más precisión la intensidad, frecuencia, tipo de consulta, duración de la misma etc. y que reflejen de forma más certera la realidad de los datos para poder tomar medidas más eficaces que propicien una cohesión social.

En la Unión Europea las medias normativas de inclusión social y digital, citadas están dando resultados aceptables aunque no homogéneos. El actual reto de la Comisión Europea con la iniciativa 2020, que fija un calendario preciso y unas metas concretas y reales, son esperanzadoras, siempre que sean apoyadas sobre todo económicamente para poder ser llevadas a cabo.

El Estado español ha propiciado una normativa de carácter general y horizontal en materia de Educación, Igualdad etc. Incluida en el Plan nacional 
de acción para la inclusión social en el Reino de España, 2008-2010 [2007], que junto con la normativa establecida ha permitido en los últimos años que generaciones de jóvenes utilicen las TIC compartiendo roles y habilidades en cuanto a su uso y frecuencia. Esta situación se distorsiona según se avanza en relación con los trabajos con los TIC, liderazgo, los altos ejecutivos, los estudios de doctorado y los puestos de responsabilidad tanto en las empresas como en las universidades, y se deben tomar medidas conectivas específicas de acuerdo con los colectivos afectados. Se debe potenciar y dotar económicamente y el Plan AVANZA2, Plan Red.es, ya que de ello depende no sólo la disminución de la brecha, sino además un crecimiento económico y una mayor cohesión social.

Las CCAA cuentan con Observatorios de la SI e iniciativas para alcanzar la inclusión digital una vez, superada la etapa de los telecentros como: hogar tic, personas mayores, asociaciones, grupos desfavorecidos, alfabetización digital para mujeres, etc. con resultados diversos. Las Comunidades Autonómicas que tienen un mayor desarrollo económico y están mejor posicionadas en el rango de acceso y utilización a Internet tienen un mayor número de planes específicos de inclusión adaptados a distintos colectivos y diversos niveles de formación. Es el caso de Madrid con el I Plan de desarrollo de la sociedad de la información y el conocimiento "Actuación en alfabetización tecnológica” http://www.madrid.org/cpresidencia/madrid_comunidad_digital/pdf, y en Cataluña el Plan Director de Infraestructuras de Telecomunicaciones (PDIT) que contempla el plan TIC como medio para lograr la igualdad, GENCAT www.gencat.net, y en las Islas Baleares destaca un ambicioso Plan director sectorial de telecomunicación http://dgtic.caib.es/www/plasectorial_telecomunicacions/extendida/pla_dgtic.

También Asturias cuenta con un Plan de acción sugerente y actualizado "Mujer piensa en TIC", dirigido a asociaciones de mujeres y profesionales, que está teniendo muy buenos resultados. Éste realiza en colaboración con el 1 Plan Avanza2, cual incluye además de un observatorio de TIC, una armonización de indicadores y la evaluación del impacto de las actuaciones, factores que influyen en su crecimiento; www.planavanza.es/informaciongeneral/.../ asturias/1_a_asturias.pdf. Aragón se decanta por una oferta selectiva en materia de la Sociedad de la Información en ayudas en materia de TIC, información sobre ¿cómo incorporar las TIC a tu negocio, quién te puede asesorar? En educación la oferta se dirige a toda la comunidad educativa; ver www.aragontic.com. El reto de las regiones tiene planes más convecionales que han obtenido resultados irregulares según el presupuesto económico anual.

Por otra parte a lo largo del estudio hemos comprobado que existen grupos meta-excluidos a los que la mayoría de estas medidas de inclusión no los 
alcanza, y a los que también se añaden otros elementos de exclusión: discapacitados, reclusos, personas mayores, etc., quienes merecen una atención muy específica si bien no son objeto de la presente investigación.

Finalmente la investigación nos permite recomendar que los poderes públicos nacionales y regionales deben invertir económicamente en planes y acciones y que, no obstante la grave situación económica por la que atravesamos, deben profundizar y desarrollar más agresivamente las medidas actuales y establecer nuevos planes de inclusión digital específicos dirigidos a diversos colectivos, que nos permitan converger con la UE y crecer en e-igual de género y de ese modo disminuir la brecha social/ digital y contribuir a una cohesión social real.

Cabe añadir que la evolución tecnológica y la incorporación de la tecnología móvil, tiene una tecnología "aliada" en las mujeres, que ya se manifiesta esperanzadora para toda la ciudadanía, que en particulas está siendo muy bien aceptada por las mujeres, nos referimos a la tecnología móvil de 3G y 4G [UIT, 2010] ya que entre otras ventajas permitirá la conciliación familiar, laboral etc. tan importante para todos.

\section{REFERENCIAS}

Brynin, M.(2006), "Gender equality through computerisation", en European sociological Review, 22, 2 pp 111-123.

Caridad, M.; Ayuso Sanchez, M. J. y Ayuso García, M. D. (2010), "Estudio comparado de la brecha digital en los países de la Unión Europea y en España (2004-2008)", en Incluçao Social. Vol.3, núm. 2, 2010, pp 54-68.

Castaño, C. (coord.) (2008), La segunda brecha digital, Madrid: Ediciones Cátedra, Universitat de Valencia, Instituto de la mujer.

Castaño, M., Martín, J. Vázquez, S. y Martínez, J. C. (2010), La brecha digital de Género. Amantes y distantes, Madrid: Universidad Complutense de Madrid.

Comisión Europea. Comunicación de la Comisión (2010). Europa 2020, Una estrategia para un crecimiento inteligente, sostenible e integrador, (En línea) Comisión Europea. COM (2010) 2020 final, Bruselas, 42p, disponible: http://ec.europa.eu/commission_2010 -2014/president/news/documents/ pdf/20100303_1_es.pdf, consultado: 3-2-2011.

(2010), Agenda Digital para Europa. COM (2010) 245 final, Bruselas, 19 de mayo de 2010, 42p, disponible: http://ec.europa. eu/information_society/digital-agenda/documents/digital-agenda-communication-en.pdf, consultado: 8-9-9. 
(2007), Iniciativa Europea 2010 para la inclusión digital «Participar en la sociedad de la información» Bruselas: COM(2007) 694final.

EUROSTAT (2010), Information society statistics: tables and figures, disponible: (http://epp.eurostat.ec.europa.eu/statistics_explained/index.php/Information_society_statistics). Consultado: 11$1-2011$

Fundación Orange (2010), Informe anual sobre el desarrollo de la SI en España 2010 (en línea), disponible: http://actualidad.orange. es/ciencia/espana-alcanza-media-europea-en-sociedad-informacion-pese-no-cumplir-objetivos-lisboa.html, consultado: 10-82010.

(2011), Informe anual sobre el desarrollo de la SI en España 2010 (en línea), disponible: http://actualidad.orange.es/ciencia/espana-alcanza-media-europea-en-sociedad-informacion-pese-no-cumplir-objetivos-lisboa.html, consultado: 3-2-2011.

Gobierno de España (2007), Ley Orgánica 3/2007 para la igualdad efectiva de hombres y mujeres de 23 de Marzo de 2007, BOE, núm. 71, pp 12611-12645.

Hafkin, N. y Huyer, S. (ed.), (2006), Cinderella or Cyber Elle? empowering women in the knowledge society, Blomfield (CT): Kumarian Press.

Instituto Nacional de Estadística (2011), Anuario Estadistico de España 2011(2011)..Madrid:INE,(en linea), disponible http://www. ine.es/prodyser/pubweb/anuarios_mnu.htm, consultado: 3-22011.

Instituto Nacional de Estadística y Ministerio de Igualdad (2011), Mujeres y Hombres en España, Madrid: INE y $\mathrm{M}^{\mathrm{o}}$ Igualda, (en linea), disponible http://www.ine.es/ss Satellite?L=0\&c=INEPub licacion_C\&cid=1259924822888\&p=1254735110672\&pagenam e=ProductosYServicios $\% 2$ FPYSLayout\&param1=PYSDetalleG ratuitas \&rendermode= previewnoinsite, consultado: 3-2-2011.

Korupp, S. y Szydlik, M. (2005), "Causes and tens of the digital divide”, en European sociological Review, 21, 4, pp. 409-422.

Ministerio de Educación, Política Social y Deportes. (2007-08), Plan nacional de acción para la inclusión social en el reino de España,2008-2010, Madrid: Mº Educación política social y Deportes.

OCDE (2006), Information technology Outlook, París: OCDE, - ICTs and gender, (2007), (en línea) DSTI/ICCP/IE (206) 9/ Final, París: OCDE, 2007, disponible:http://www.oecd.org/document/0,3746, en_2649_201185_46462759_1_1_1_1,00, consultado:20-9-2010.

Pimienta, D. (2008), Brecha digital, brecha social, brecha paradigmática, II Coloquio Internacional E- DOCPA, Oviedo: Consejería de Administraciones Públicas 
Plan Avanza 2, Estrategia 2011-2015, Plan Avanza 2, Medidas de impulso a la SI, (2010) Consejo de Ministros de 16 de julio de 2010 (en línea), disponible:http://www.planavanza.es/InformacionGeneral/Estrategia2011/Paginas/Estrategia2011_2015.aspx

EUROSTAT (2010), information society statistics: tables and figures, disponible: (http://epp.eurostat.ec.europa.eu/statistics_explai ned/index.php/Information_society_statistics), consultado: 11-12011.

Van Welsum, D. y Montaigner, P. (2007), ICTs and gender: working party on the information economy, París: OECD.

Unión Internacional de Telecomunicaciones, ITU (2010), Medición de la Sociedad de la Información, Ginebra: Unión Internacional de Telecomunicaciones, (en línea), disponible: www.itu.int/es, consultado: 10-2-11.

Wavermans, L. et al. (2010), The connectivity Scorecard 2010. Report LEGG Nokia Siemens networks, (en línea) 61p, disponible: http:// www.connectivityscorecard.org/images/uplmoads/media/The_ Connectivity Report2010.pdf, consultado: 5-2-2010. 
ANEXO 1

\begin{tabular}{|c|c|c|c|c|c|}
\hline \multirow{2}{*}{$\begin{array}{l}\text { Comunidades } \\
\text { Autónomas }\end{array}$} & \multicolumn{2}{|c|}{ Plan esp. TIC } & \multicolumn{2}{|c|}{ Nivel que ofrece } & \multirow{2}{*}{$\begin{array}{c}\text { Alfabetización } \\
\text { tecnológica }\end{array}$} \\
\hline & $\mathrm{Si}$ & No & Básico & Avanzado & \\
\hline Andalucía & $x$ & & & $x$ & Alfabetización TIC mujeres \\
\hline Aragón & $x$ & & $x$ & & $\begin{array}{l}\text { Teruel digital colectivo amas de } \\
\text { casa etc. }\end{array}$ \\
\hline Asturias & $x$ & & & $x$ & $\begin{array}{l}\text { "Mujer piensa en Tl.. C } \\
\text { asociaciones y profesionales }\end{array}$ \\
\hline Baleares & & $x$ & $x$ & $x$ & $\begin{array}{l}\text { Plan director sector } \\
\text { telecomunicaciones }\end{array}$ \\
\hline Canarias & & $x$ & $x$ & $x$ & Plan Avanza 2 (No específico) \\
\hline Cantabria & & $x$ & $x$ & & - - \\
\hline Castilla Mancha & & $x$ & $x$ & & Incluido bienestar social \\
\hline Castilla León & $x$ & & $x$ & & $\begin{array}{l}\text { TIC como herramienta para } \\
\text { igualdad }\end{array}$ \\
\hline Cataluña & $x$ & & $x$ & $x$ & $\begin{array}{l}\text { TIC como medio para igualdad } \\
\text { GESCAT }\end{array}$ \\
\hline C Valenciana & $x$ & & $x$ & & Modelo SI incluyente PETIC \\
\hline Extremadura & & $x$ & $x$ & $x$ & Desarrollo de las TIC para todos \\
\hline Galicia & $x$ & & $x$ & $x$ & $\begin{array}{l}\text { Empresarias. Amas de casa. } \\
\text { Profesionales }\end{array}$ \\
\hline Rioja & $x$ & & $x$ & & Alfabetización tecnológica \\
\hline C Madrid & $x$ & & $x$ & $x$ & $\begin{array}{l}\text { Actuación en alfabetización } \\
\text { tecnológica }\end{array}$ \\
\hline C R Murcia & & $x$ & $x$ & & $\begin{array}{l}\text { Planes para el desarrollo SI en } \\
\text { Murcia }\end{array}$ \\
\hline C F Navarra & $x$ & & $x$ & & $\begin{array}{l}\text { Plan formación TIC a mujeres } \\
\text { proceso exclusión digital }\end{array}$ \\
\hline País Vasco & $x$ & & $x$ & & $\begin{array}{l}\text { Alfabetización mujeres riesgo } \\
\text { exclusión digital }\end{array}$ \\
\hline
\end{tabular}

